\title{
Impacts of Patient Characteristics on the Effectiveness of Landiolol in AF/AFL Patients Complicated with LV Dysfunction: Subgroup Analysis of the J-Land Study
}

\author{
Koichiro Kinugawa $\cdot$ Ryozo Nagai $\cdot$ Hiroshi Inoue $\cdot$ Hirotsugu Atarashi $\cdot$ Yoshihiko Seino $\cdot$ \\ Takeshi Yamashita $\cdot$ Wataru Shimizu $\cdot$ Takeshi Aiba $\cdot$ Masafumi Kitakaze $\cdot$ Atsuhiro Sakamoto \\ Takanori Ikeda $\cdot$ Yasushi Imai · Takashi Daimon $\cdot$ Katsuhiro Fujino • Tetsuji Nagano • \\ Tatsuaki Okamura $\cdot$ Masatsugu Hori $\cdot$ The J-Land Investigators \\ To view enhanced content go to www.advancesintherapy.com \\ Received: January 16, 2014 / Published online: March 19, 2014 \\ (c) The Author(s) 2014. This article is published with open access at Springerlink.com
}

\section{ABSTRACT}

Introduction: Results from the multicenter trial (J-Land study) of landiolol versus digoxin in atrial fibrillation (AF) and atrial flutter (AFL) patients with left ventricular (LV) dysfunction

Clinical Trials Registration: JapicCTI-111,448.

Electronic supplementary material The online version of this article (doi:10.1007/s12325-014-0111-2) contains supplementary material, which is available to authorized users.

K. Kinugawa $(\square)$

Department of Therapeutic Strategy for Heart Failure, Graduate School of Medicine, University of Tokyo, 7-3-1 Hongo, Bunkyo-ku, Tokyo 113-8655, Japan

e-mail: kinugawa-tky@umin.ac.jp

R. Nagai

Jichi Medical University, Shimotsuke, Japan

H. Inoue

The Second Department of Internal Medicine,

Graduate School of Medicine, University of Toyama,

Toyama, Japan

H. Atarashi

Department of Internal Medicine, Nippon Medical

School Tama-Nagayama Hospital, Tokyo, Japan

Y. Seino

Department of Cardiology, Nippon Medical School

Chiba-Hokusoh Hospital, Chiba, Japan revealed that landiolol was more effective for controlling rapid HR than digoxin. The subgroup analysis for patient characteristics was conducted to evaluate the impact on the efficacy and safety of landiolol compared with digoxin.

Methods: Two hundred patients with AF/AFL, heart rate $(\mathrm{HR}) \geq 120$ beats/min, and $\mathrm{LV}$ ejection fraction (LVEF) 25-50\% were randomized to receive either landiolol $(n=93)$ or digoxin $(n=107)$. Successful HR control was defined as $\geq 20 \%$ reduction in HR together with

T. Yamashita

The Cardiovascular Institute, Tokyo, Japan

W. Shimizu

Department of Cardiovascular Medicine, Nippon

Medical School, Tokyo, Japan

T. Aiba $\cdot$ M. Kitakaze

Department of Cardiovascular Medicine, National Cerebral and Cardiovascular Center, Suita, Japan

A. Sakamoto

Department of Anesthesiology, Nippon Medical

School, Tokyo, Japan

T. Ikeda

Department of Cardiovascular Medicine, Toho

University Faculty of Medicine, Tokyo, Japan

Y. Imai

Department of Cardiovascular Medicine, Jichi

Medical University, Shimotsuke, Japan 
$\mathrm{HR}<110$ beats/min at $2 \mathrm{~h}$ after starting intravenous administration of landiolol or digoxin. The subgroup analysis for patient characteristics was to evaluate the impact on the effectiveness of landiolol in AF/AFL patients complicated with LV dysfunction.

Results: The efficacy in patients with NYHA class III/NYHA class IV was $52.3 \% / 35.3 \%$ in landiolol, and $13.8 \% / 9.1 \%$ in digoxin $(p<0.001$ and $p=0.172)$, lower LVEF (25-35\%)/higher LVEF (35-50\%) was $45.7 \% / 51.1 \%$ in landiolol, and $14.0 \% / 12.7 \%$ in digoxin $(p<0.001$ and $p<0.001)$, CKD stage $1(90<\mathrm{eGFR}) / \mathrm{CKD}$ stage $2 \quad(60 \leq \mathrm{eGFR}<90) / \mathrm{CKD} \quad$ stage 3 $(30 \leq$ eGFR $<60) /$ CKD stage $4(15 \leq$ eGFR $<30)$ was $66.7 \% / 59.1 \% / 39.6 \% / 66.7 \%$ in landiolol, and $0 \% / 13.8 \% / 17.0 \% / 0 \%$ in digoxin $(p=0.003, p<0.001, p=0.015$ and $p=0.040)$. Conclusions: This subgroup analysis indicated that landiolol was more useful, regardless of patient characteristics, as compared with digoxin in AF/AFL patients complicated with LV dysfunction. Particularly, in patients with impaired renal function, landiolol should be preferred for the purpose of acute rate control of AF/AFL tachycardia.

Keywords: Landiolol; Digoxin; Rate control; Acute; Japanese; Atrial fibrillation; Atrial flutter; Left ventricular dysfunction; Heart failure; J-Land

T. Daimon

Department of Biostatistics, Hyogo College of

Medicine, Nishinomiya, Japan

K. Fujino · T. Nagano · T. Okamura

Ono Pharmaceutical Co. Ltd, Osaka, Japan

T. Okamura

e-mail: t.okamura@ono.co.jp

M. Hori

Osaka Medical Center for Cancer and

Cardiovascular Diseases, Osaka, Japan

\section{INTRODUCTION}

Atrial fibrillation (AF) and atrial flutter (AFL) are common arrhythmias in patients with left ventricular (LV) dysfunction. Over 20\% of patients with heart failure exhibit AF $[1,2]$. In these patients, AF/AFL are often associated with a rapid ventricular response during the worsening of heart failure [3, 4]. However, a sustained rapid ventricular response may further deteriorate cardiac function [5], accelerating the symptoms of heart failure [68]. Intravenous administration of digoxin is considered the standard therapy for controlling the rapid ventricular response in AF/AFL patients with cardiac dysfunction or heart failure [4, 9]. Although digoxin has some beneficial effects for treating heart failure by way of its positive inotropic effects, digoxin may also have a negative chronotropic effect as a result of vagal stimulation. Of note, the negative chronotropic effect develops much more slowly, often taking several hours to reach the maximal effect $[9,10]$. Short-acting parenteral $\beta$-blockers can act more rapidly than digoxin, and may provide a swift control of heart rate (HR) in these clinical settings. However, there is a concern that $\beta$-blockers may depress cardiac function and further deteriorate ventricular dysfunction, accelerating heart failure. Landiolol, an ultrashort-acting $\beta$-blocker, is rapidly metabolized to inactive forms in the blood and liver, resulting in a short half-life of approximately $4 \mathrm{~min}$ in human blood. In addition, it selectively binds to $\beta 1$ receptors, with a $\beta 1$ receptor selectivity $(\beta 1 / \beta 2)$ as high as 251 [11]. Based on these properties, landiolol has been reported to be useful for treating several acute disorders, including arrhythmias during heart surgery [12], acute myocardial infarction [13], acute decompensated heart failure [14], and refractory 
electrical storm [15]. Ultra-short-acting $\beta$ blockers may be useful to control HR with minimal effects on cardiac function. Even though the negative inotropic effect by landiolol is manifested, it is not theoretically and practically sustained by decreasing the dose or stopping administration of these drugs.

This hypothesis was tested in the Japanese Landiolol versus Digoxin study (J-Land study), which investigated the efficacy and safety of intravenous landiolol for achieving rapid control of tachycardia in patients with AF/AFL and LV dysfunction. The results of the J-Land study showed that landiolol was more effective for controlling rapid HR than digoxin in AF/AFL patients with LV dysfunction [16]. To further assess these findings, we analyzed effectiveness in the J-Land study population according to patient characteristics.

\section{METHODS}

\section{Study Design and Patients}

The design of the J-Land study has been described previously [16]. In brief, the J-Land study was a central registration, prospective, multicenter, single-blind, randomized, parallelgroup study that included 200 patients with AF/AFL and LV dysfunction. Average age was $71.6 \pm 11.5$ years, $106(53 \%)$ were men, New York Heart Association (NYHA) classes were equally distributed between III $(n=163$, $81.9 \%)$ and IV $(n=36,51 \%)$, Left ventricular ejection fraction (LVEF) was 36.6 $\pm 7.6 \%$, and heart rate $(\mathrm{HR})$ was $138.1 \pm 15.3$ beats/min in average. The use of antiarrhythmic drugs, sympathomimetic drugs, sympatholytic drugs, defibrillator use, catheter ablation, and pacemaker therapy were prohibited from the enrollment until completing all observations at $2 \mathrm{~h}$ after starting treatment. However, patients being treated with guideline-directed oral $\beta$-blockers (carvedilol or bisoprolol) or oral digitalis preparations for chronic heart failure, chronic AF, and/or chronic AFL could participate in the study under continued treatment without changes in their doses. In the landiolol group, continuous administration of landiolol was intravenously started at a dose of $1 \mu \mathrm{g} / \mathrm{kg} / \mathrm{min}$ and titrated to a maximum dose of $10 \mu \mathrm{g} / \mathrm{kg} / \mathrm{min}$ according to the patient's condition. Landiolol was administered for $\geq 2 \mathrm{~h}$ and up to $72 \mathrm{~h}$. In the digoxin group, digoxin was intravenously administered at an initial dose of $0.25 \mathrm{mg}$ and could be uptitrated within $72 \mathrm{~h}$ according to the patient's condition. The Japanese guideline for the treatment of atrial fibrillation recommends that the maximum dose of digoxin is $0.25 \mathrm{mg}$ within $2 \mathrm{~h}$. For patients treated with oral digitalis, the parenteral digoxin dose could be reduced to $0.125 \mathrm{mg}$ according to the patient's condition to prevent digitalis intoxication. The final observation of this study was performed for up to $48 \mathrm{~h}$ after the end of administration of landiolol or for up to $48 \mathrm{~h}$ after the final dose in the digoxin group. The investigators for the study are listed in the Appendix [16].

In this study, we analyzed the primary endpoint, in which the percentage of patients with both $\mathrm{HR}<110$ beats/min and $\geq 20 \%$ decrease from baseline at $2 \mathrm{~h}$ after administration. Heart rate was measured by the electrocardiogram over $1 \mathrm{~min}$ and was reviewed at the core laboratory in a blinded manner. The safety endpoint was the incidence of adverse events related or unrelated to the study drugs. For this analysis, we divided the landiolol and the digoxin groups by patient characteristics at baseline (Fig. 1). 


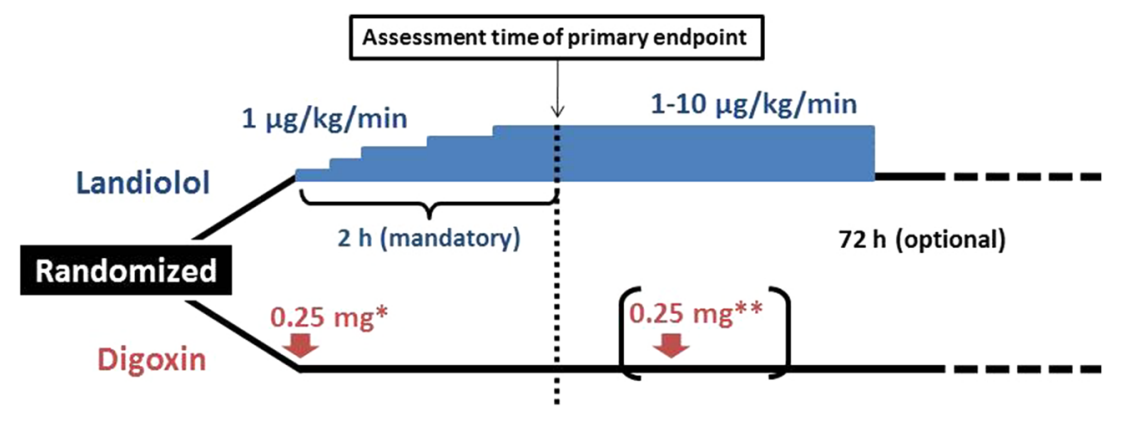

Fig. 1 Study protocol

\section{Statistical Analysis}

Data are expressed as the mean \pm standard deviation or percentages of patients. Student's $t$ test and $\chi^{2}$ test were used to compare the means and percentages, respectively, between the two groups. The subgroup analysis for the primary endpoint was conducted by stratifying the J-Land study population by age, sex, NYHA, baseline HR, baseline systolic blood pressure (SBP), LVEF, $\beta$-blocker and eGFR using a linear probability model with HR and LVEF measured immediately before starting the study drug as covariates. The subgroup analysis for adverse events was conducted by stratifying the J-Land study population by the above factors using $\chi^{2}$ test. Values of $p<0.05$ were considered statistically significant (2-sided). All analyses were performed using SAS version 9.2 for Windows (SAS Institute, Cary, NC, USA).

\section{RESULTS}

\section{Patient Disposition and Baseline Characteristics}

The disposition of patients in this study is shown in Fig. 2. A total of 214 patients were randomized to either landiolol $(n=99)$ or digoxin $(n=115)$. Of these, 14 patients were not treated (the landiolol group, $n=6$; the digoxin group, $n=8$ ) and 2 patients in the landiolol group did not comply with the protocol. Therefore, 200 patients (the landiolol group, $n=93$; the digoxin group, $n=107$ ) were included in the safety subgroup analysis set. Of these, 18 patients had no data of the primary endpoint (the landiolol group, $n=9$; the digoxin group, $n=9$ ). Therefore, 180 patients were included in the efficacy subgroup analysis set (the landiolol group, $n=82$; the digoxin group, $n=98$ ). The demographics of the study patients are shown in Table 1 . There were no differences in the general characteristics of the 2 groups. 141 patients $(70.5 \%)$ were $\geq 65$ years, and 106 patients (53.0\%) were male. The NYHA class was III in 163 patients (81.9\%) and IV in 36 patients (18.1\%). 115 patients (58.4\%) were $\mathrm{HR}<140 \mathrm{bpm}$, and 111 patients (55.8\%) were $\mathrm{SBP} \geq 120 \mathrm{mmHg}$. Before starting study treatment, oral $\beta$-blockers were used in 41 patients (20.5\%). About $60 \%$ of patients or more had moderate or severe renal dysfunction $\quad(15 \leq$ eGFR $\quad(\mathrm{mL} / \mathrm{min} /$ $\left.1.73 \mathrm{~m}^{2}\right)<60$ ).

\section{Efficacy}

The subgroup analysis of the primary endpoint is shown in Table 2 . The percentage of patients with both $\mathrm{HR}<110$ beats/min and $\geq 20 \%$ decrease from baseline to $2 \mathrm{~h}$ after administration was determined to examine the influence of HR and LVEF at baseline. Overall, 


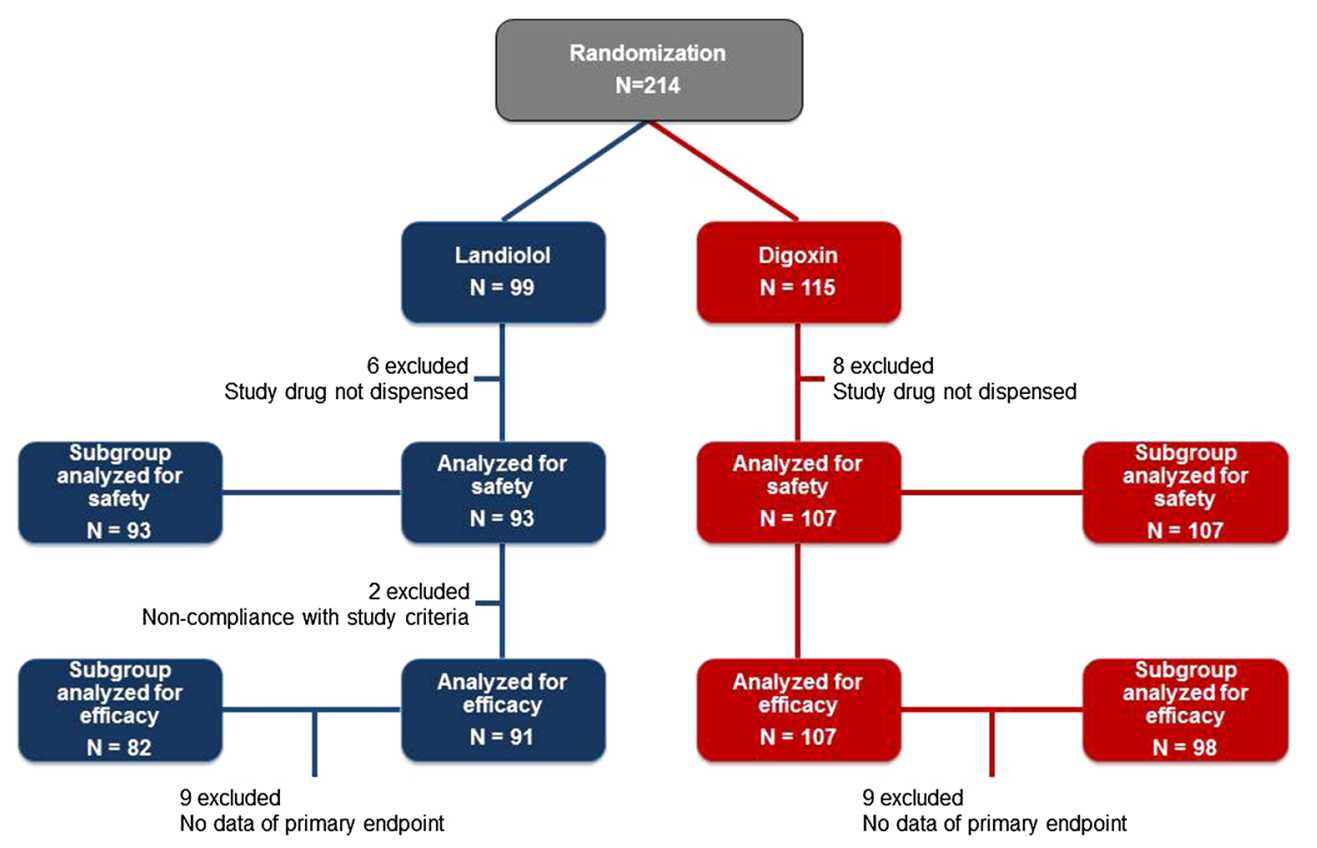

Fig. 2 Patient disposition

$48.0 \%(n=40 / 82)$ of patients in the landiolol group and $13.9 \%(n=13 / 98)$ of patients in the digoxin group achieved the primary endpoint, with a between-group difference of $34.1 \%$ (95\% confidence interval, 22.1-46.2; $p<0.0001$ ). There was no impact on the primary endpoint by age, sex, baseline SBP, LVEF, and $\beta$-blocker (oral) intake. In patients with NYHA IV, there was no significant difference between the two groups, but the percentage of patients who reached the primary endpoint was numerically higher in the landiolol group [the landiolol group; $35.3 \%$ (6/17 patients), and the digoxin group; $9.1 \%$ (1/11 patients)]. In patients with renal dysfunction of moderate or more [15 $\leq$ eGFR $\left.\quad\left(\mathrm{mL} / \mathrm{min} / 1.73 \mathrm{~m}^{2}\right)<60\right]$, the landiolol group had also a significantly better outcome as compared to the digoxin group.

\section{Safety}

The incidence of the adverse events in the subgroup is shown in Table 3 . Adverse events occurred in 30 patients $(32.3 \%)$ in the landiolol group and in 35 patients (32.7\%) in the digoxin group, which was not statistically significant $(p=0.946)$. There was no impact on the incidence of the adverse events in the subgroup by age, sex, NYHA, baseline HR, LVEF, and oral $\beta$-blocker intake. In the population with low SBP, the incidence of the adverse events was significantly higher in the digoxin group compared with the landiolol group. Conversely, in the population with high SBP, the incidence of adverse events was significantly higher in the landiolol group compared with the digoxin group. Adverse events associated with heart and renal function are shown in Table 4 . In the safety subgroup analysis of SBP, the incidence of adverse events associated with heart and renal function was $12.8 \%(5 / 39)$ in the landiolol group with low SBP, $13.2 \%(7 / 53)$ in the landiolol group with high SBP, 24.5\% (12/49) in the digoxin group with low SBP, and 6.9\% (4/ 58 ) in the digoxin group with high SBP. In the 
Table 1 Baseline characteristics

\begin{tabular}{|c|c|c|c|c|}
\hline & Total & Landiolol & Digoxin & $p$ value \\
\hline Age $(n)$ & & 93 & 107 & \\
\hline 20 to $<65$ years & $59(29.5 \%)$ & $32(34.4 \%)$ & $27(25.2 \%)$ & \\
\hline$\geq 65$ years & $141(70.5 \%)$ & $61(65.6 \%)$ & $80(74.8 \%)$ & \\
\hline Mean $\pm S D$ & $71.6 \pm 11.5$ & $70.5 \pm 12.0$ & $72.5 \pm 11.0$ & 0.221 \\
\hline $\operatorname{Sex}(n)$ & & 93 & 107 & \\
\hline Male & $106(53.0 \%)$ & $50(53.8 \%)$ & $56(52.3 \%)$ & 0.840 \\
\hline Female & $94(47.0 \%)$ & $43(46.2 \%)$ & $51(47.7 \%)$ & \\
\hline NYHA $(n)$ & & 93 & 107 & \\
\hline Class III & $163(81.9 \%)$ & $71(77.2 \%)$ & $92(86.0 \%)$ & 0.108 \\
\hline Class IV & $36(18.1 \%)$ & $21(22.8 \%)$ & $15(14.0 \%)$ & \\
\hline Baseline HR $(n)$ & & 90 & 107 & \\
\hline$<140 \mathrm{bpm}$ & $115(58.4 \%)$ & $55(61.1 \%)$ & $60(56.1 \%)$ & \\
\hline$\geq 140 \mathrm{bpm}$ & $82(41.6 \%)$ & $35(38.9 \%)$ & $47(43.9 \%)$ & \\
\hline Mean $\pm S D$ & $138.1 \pm 15.3$ & $138.2 \pm 15.7$ & $138.0 \pm 15.0$ & 0.934 \\
\hline Baseline SBP $(n)$ & & 92 & 107 & \\
\hline$<120 \mathrm{mmHg}$ & $88(44.2 \%)$ & $39(42.4 \%)$ & $49(45.8 \%)$ & \\
\hline$\geq 120 \mathrm{mmHg}$ & $111(55.8 \%)$ & $53(57.6 \%)$ & $58(54.2 \%)$ & \\
\hline Mean $\pm S D$ & $125.7 \pm 21.8$ & $124.6 \pm 19.8$ & $126.6+23.5$ & 0.523 \\
\hline $\operatorname{LVEF}(n)$ & & 92 & 107 & \\
\hline 25.0 to $<35.0 \%$ & $90(45.2 \%)$ & $41(44.6 \%)$ & $49(45.8 \%)$ & \\
\hline 35.0 to $50.0 \%$ & $109(54.8 \%)$ & $51(55.4 \%)$ & $58(54.2 \%)$ & \\
\hline Mean $\pm S D$ & $36.6 \pm 7.6$ & $36.4 \pm 7.9$ & $36.7 \pm 7.3$ & 0.753 \\
\hline Beta blocker (oral) $(n)$ & & 93 & 107 & \\
\hline No beta-blockers intake & $159(79.5 \%)$ & $75(80.6 \%)$ & $84(78.5 \%)$ & \\
\hline Beta-blockers intake & $41(20.5 \%)$ & $18(19.4 \%)$ & $23(21.5 \%)$ & 0.708 \\
\hline $\operatorname{eGFR}(n)$ & & 92 & 107 & \\
\hline $90 \leq \mathrm{eGFR}$ & $18(9.1 \%)$ & $8(8.7 \%)$ & $10(9.4 \%)$ & \\
\hline $60 \leq \mathrm{eGFR}<90$ & $58(29.1 \%)$ & $26(28.3 \%)$ & $32(29.9 \%)$ & \\
\hline $30 \leq \mathrm{eGFR}<60$ & $109(54.8 \%)$ & $51(55.4 \%)$ & $58(54.2 \%)$ & \\
\hline $15 \leq \mathrm{eGFR}<30$ & $14(7.0 \%)$ & $7(7.6 \%)$ & $7(6.5 \%)$ & \\
\hline Mean \pm SD & $57.3 \pm 19.7$ & $57.6 \pm 19.7$ & $57.0 \pm 19.8$ & 0.845 \\
\hline
\end{tabular}

Mean \pm SD or number (\%), Student's $t$ test and $\chi^{2}$ test 
digoxin group with low SBP, the incidence of adverse events associated with heart and renal function was significantly higher as compared to the high SBP group $(p=0.011)$. In addition, in patients with severe renal impairment [15 $\leq$ eGFR $\left.\quad\left(\mathrm{mL} / \mathrm{min} / 1.73 \mathrm{~m}^{2}\right)<30\right], \quad$ the incidence of adverse events was significantly lower in the landiolol group than compared with the digoxin group.

\section{DISCUSSION}

Our results showed that in patients with LV dysfunction, who had AF/AFL with HR of $120 \mathrm{bpm}$ or higher, there was a subgroup that the administration of landiolol should be recommended. Our subgroup analysis in patients with severe renal dysfunction demonstrated that the incidence of adverse events was significantly low in the landiolol group compared with the digoxin group, and that swift rate control effect was significantly more prevalent by landiolol than by digoxin.

According to the several registries for chronic heart failure patients, the ratio of patients with renal impairment has been reported approximately to be 30-70\% (ADHERE, JCARECARD, CHART) [18-20]. It is also reported that long-term prognosis is poor if renal dysfunction is severe [19]. In the treatment of acute heart failure, there have been concerns about nesiritide that may deteriorate renal function

Table 2 Subgroup analysis for primary endpoint

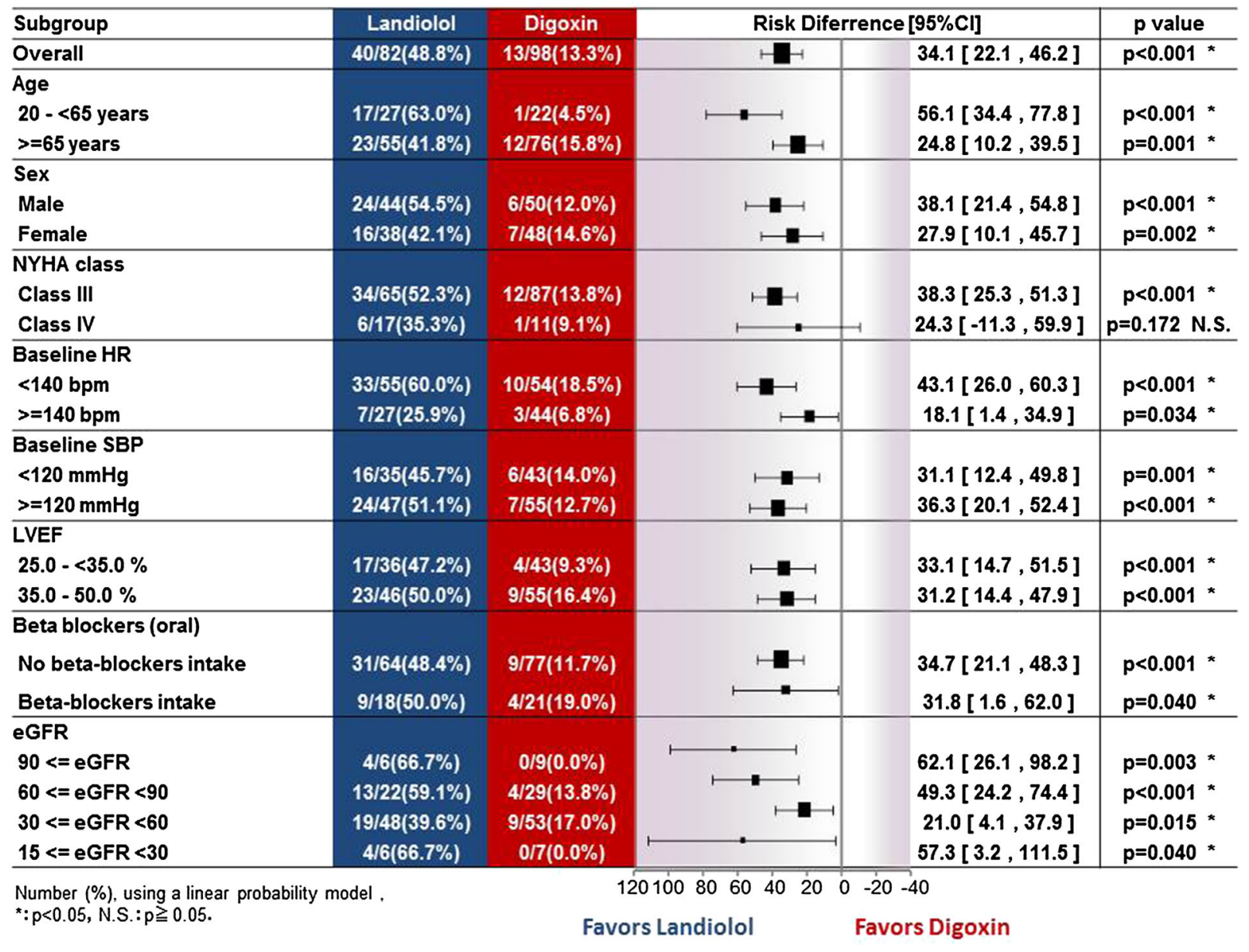


Table 3 Subgroup analysis for adverse events

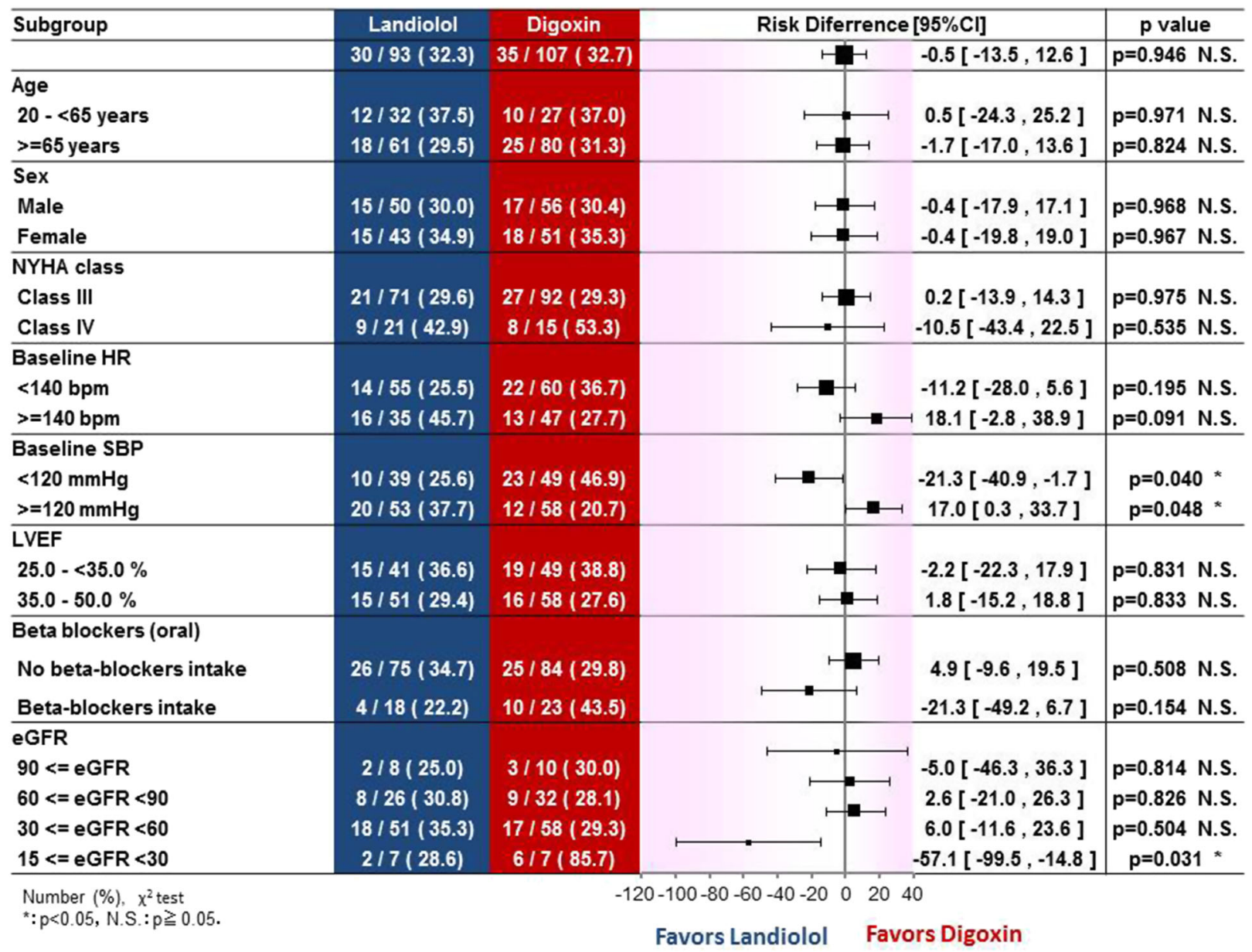

and worsen short-term prognosis (30 days after) $[21,22]$. ASCEND-HF study never proved any significant efficacy over placebo, which resulted in a marked decline in the share of nesiritide [23]. On the other hand, serelaxin, which is currently being developed as a therapeutic agent for acute heart failure, improved the prognosis after 180 days of administration with the fewer adverse events related to renal function [24, 25]. Accordingly, safety for renal function may be indispensable for less adverse impact on the long-term prognosis of acute heart failure patients.

Digoxin is excreted by kidneys and its dose should be decreased in many patients with renal dysfunction [17]. However, landiolol is not metabolized at all by kidneys or neither have adverse effects on renal function. As a result, landiolol can be uptitrated safely to achieve rate control in a shorter period regardless of patients' renal function. In fact, landiolol was faster than digoxin in the rate control during acute phase of AF/AFL among the patients with renal dysfunction. Moreover, our subgroup analysis consistently demonstrated that the incidence of adverse events was lower by the landiolol treatment compared with the digoxin in patients with severe renal dysfunction. Furthermore, in the digoxin group with low SBP, that is considered to have decreased renal 


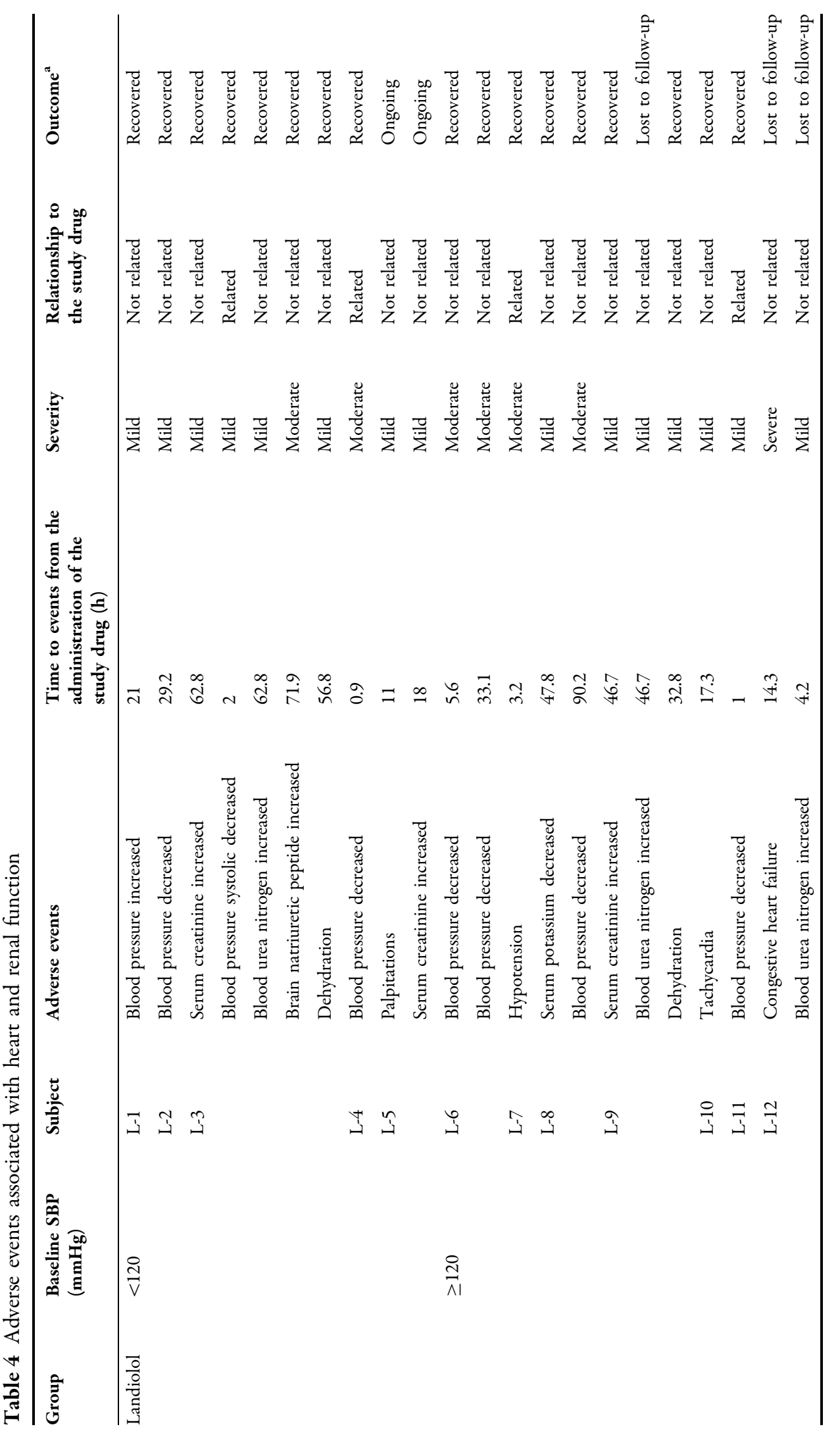




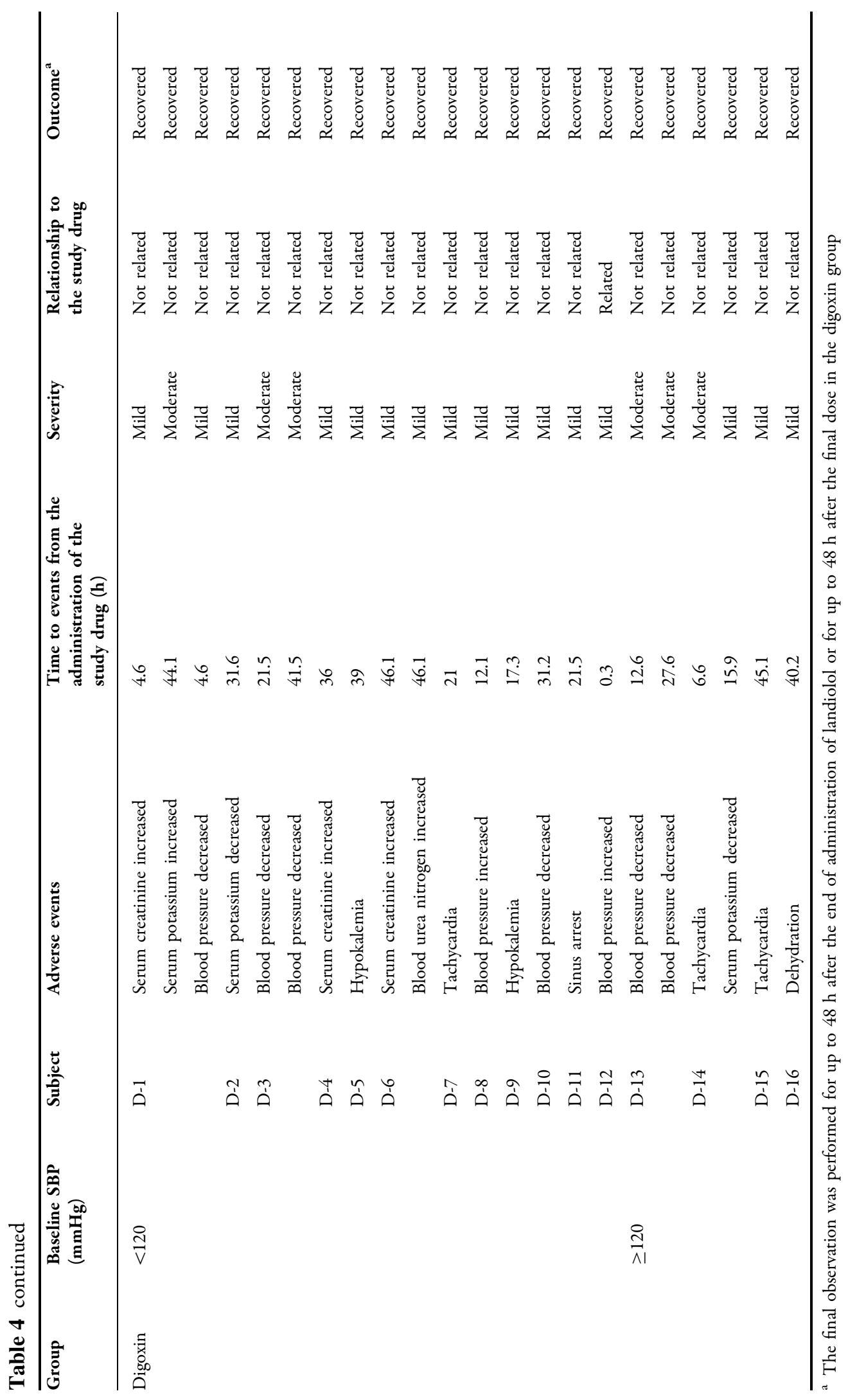


blood flow, the incidence of adverse events related to heart and renal function has increased. We speculate the delayed excretion of digoxin due to a decrease in renal blood flow has affected the incidence of adverse events. In this regard, for the rate control during acute phase of AF/AFL patients, especially those who are associated with severe renal dysfunction, landiolol should be considered as the firstchoice drug.

This study had several limitations. Firstly, the efficacy of landiolol in patients in cardiogenic shock was not examined because patients with $\mathrm{SBP}<90 \mathrm{mmHg}$ were excluded. Secondly, the efficacy of landiolol in patients with severe LV dysfunction was not determined because patients with a baseline LVEF $<25 \%$ were excluded. Thirdly, the impact of landiolol on the long-term prognosis should be examined in the future. Lastly, in this study, the efficacy and safety was only compared between landiolol and digoxin. Therefore, it is still unclear whether the effects of landiolol are superior to those of propranolol or esmolol. However, the $t 1 / 2$ of landiolol is shorter than that of propranolol and esmolol [26], and $\beta 1$ selectivity is higher [27], which make landiolol favorable for use in the acute rate control of $\mathrm{AF} /$ AFL tachycardia because of less possibility of serious adverse events.

\section{CONCLUSIONS}

This subgroup analysis indicated that landiolol was more useful, regardless of patient characteristics, as compared with digoxin in AF/AFL patients complicated with LV dysfunction. Particularly, in the rate control of AF/AFL tachycardia patients with impaired renal function, landiolol should be preferred over digoxin.

\section{ACKNOWLEDGMENTS}

The authors thank all of the investigators and site staff who participated in this clinical trial.

Conflict of interest. This study was funded by Ono Pharmaceutical Co Ltd (Osaka, Japan). K.K., R.N., H.I., H.A., Y.S., T.Y., W.S., T.A., M.K., A.S., T.I., Y.I., T.D., and M.H. have received consulting fees from Ono Pharmaceutical. K.F., T.N., and T.O. are employees of Ono Pharmaceutical.

Open Access. This article is distributed under the terms of the Creative Commons Attribution Noncommercial License which permits any noncommercial use, distribution, and reproduction in any medium, provided the original author(s) and the source are credited.

\section{APPENDIX}

\section{Investigators and Study Sites}

Toshiharu Takeuchi, Asahikawa Medical University Hospital; Shigeo Kakinoki, Otaru Kyokai Hospital; Minoru Sato, Hokkaido Medical Center; Hironori Murakami, Teine Keijinkai Hospital; Ken Okumura, Hirosaki University Graduate School of Medicine; Tetsuo Yagi, Sendai City Hospital; Tsuyoshi Shinozaki, Sendai Medical Center; Koji Fukuda, Tohoku University Graduate School of Medicine; Kaname Takizawa, Sendai Kousei Hospital; Tetsu Watanabe, Yamagata University Hospital; Shuichi Taguchi, Mito Medical Center; Shoji Suzuki, Kasumigaura Medical Center; Kazutaka Aonuma, Tsukuba University Hospital; Daisuke Abe, Ibaraki Prefectural Central Hospital; Shoichi Tange, Maebashi Red Cross Hospital; Shigeto Naito, 
Gunma Prefectural Cardiovascular Center; Shu Kasama, Cardiovascular Hospital of Central Japan (Kitakanto Cardiovascular Hospital); Shin-ichi Momomura, Saitama Medical Center, Jichi Medical University; Kazuo Matsumoto, Saitama Medical University International Medical Center; Masayuki Inagaki, Funabashi Municipal Medical Center; Atsushi Iwasa, New Tokyo Hospital; Yoshihiko Seino, Nippon Medical School Chiba-Hokusoh Hospital; Atsushi Hirayama, Nihon University School of Medicine, Itabashi Hospital; Takanori Ikeda, Toho University Omori Medical Center; Seiji Fukamizu, Tokyo Metropolitan Hiroo Hospital; Jun Umemura, Sakakibara Heart Institute; Hiroyuki Niinuma, St. Luke's International Hospital; Koichiro Kinugawa, Tokyo University Hospital; Kaoru Sugi, Toho University Ohashi Medical Center; Hiroyuki Tanaka, Tokyo Metropolitan Tama Medical Center; Yoshiki Kusama, Nippon Medical School TamaNagayama Hospital; Atsuyuki Ono, Kasai Shoikai Hospital; Takeshi Yamashita, The Cardiovascular Institute; Kazunori Iwade, National Hospital Organization Yokohama Medical Center; Kazuo Kimura, Yokohama City University Medical Center; Hiroshi Suzuki, Showa University Fujigaoka Hospital; Hideo Himeno, Fujisawa City Hospital; Koichiro Yoshioka, Tokai University Hospital; Hiroshi Furushima, Niigata University Graduate School of Medical and Dental Sciences; Hirotaka Oda, Niigata City General Hospital; Koichi Fuse, Tachikawa General Hospital; Hiroshi Inoue, Toyama University Hospital; Tetsuo Konno, Kanazawa University Hospital; Masayuki Takamura, Kanazawa University Hospital; Kenji Sakata, Kanazawa Cardiovascular Hospital; Ken Umetani, Yamanashi Prefectural Central Hospital; Kenichi Kawabata, Yamanashi University Hospital; Hiroshi Tsutsui, Japanese Red Cross Society Suwa Hospital; Masahiro
Muto, Hamamatsu Medical Center; Yasushi Wakabayashi, Seirei Mikatahara General Hospital; Yasuya Inden, Nagoya University Hospital; Hiroaki Sano, Nagoya Ekisaikai Hospital; Haruo Kamiya, Japanese Red Cross Nagoya Daiichi Hospital; Toshikazu Tanaka, Okazaki City Hospital; Masayoshi Ajioka, Tosei General Hospital; Yukio Ozaki, Fujita Health University Hospital; Tetsuya Amano, Aichi Medical University Hospital; Makoto Kitamuta, Kyoto Second Red Cross Hospital; Masahiro Esato, Ijinkai Takeda General Hospital; Kimihito Usui, Maizuru Mutual Hospital; Eiwa Zen, Koseikai Takeda Hospital; Kenshi Fujii, Sakurabashi Watanabe Hospital; Takahisa Yamada, Osaka General Medical Center; Yasushi Sakata, Osaka University Hospital; Yoshiyuki Nagai, Rinku General Medical Center; Shiro Kamakura, National Cerebral and Cardiovascular Center; Takashi Kurita, Kinki University Hospital; Akihiko Takahashi, Sakurakai Takahashi Hospital; Toshiro Shinke, Kobe University Hospital; Kazuyasu Yoshitani, Hyogo Prefectural Amagasaki Hospital; Takatoshi Hayashi, Hyogo Brain and Heart Center; Yorihiko Higashino, Higashi Takarazuka Satoh Hospital; Koichi Tamita, Nishinomiya Watanabe Cardiovascular Center; Satoshi Nagase, Okayama University Hospital; Ritsu Tamura, National Hospital Organization Kure Medical Center; Kaoru Yanagihara, Higashihiroshima Medical Center; Nobuo Shiode, Tsuchiya General Hospital; Yuji Shimatani, Hiroshima City Hospital; Mitsunori Okamoto, Hiroshima Prefectural Hospital; Shigeki Kobayashi, Yamaguchi University Hospital; Shinobu Hosokawa, Tokushima Red Cross Hospital; Eitaro Umehara, Kaisei Hospital; Mitsunori Abe, Yotsuba Circulation Clinic; Masahiko Goya, Kokura Memorial Hospital; Toshiaki Kadokami, Saiseikai Futsukaichi Hospital; Yusuke Yamamoto, Saiseikai Fukuoka 
General Hospital; Keijiro Saku, Fukuoka University Hospital; Ryozo Kobayashi, National Hospital Organization Kyusyu Medical Center; Tomohiro Kawasaki, ShinKoga Hospital; Hiroshige Yamabe, Kumamoto University Hospital; Kazuhiro Nishigami, Saiseikai Kumamoto Hospital Cardiovascular Center; Shunichi Koide, Yatsushiro Social Insurance General Hospital; Masayuki Kaneko, Oita Oka Hospital; Masahiro Sonoda, National Hospital Organization Kagoshima Medical Center; Tetsuji Shinjyo, Tomishiro Central Hospital.

\section{Ono Pharmaceutical Core Team}

Takashi Tanaka, Takuto Kuramoto, Taketo Anze, Nobuyuki Oki (data manager), Akira Tsuchiya (data manager), Keita Nagasawa (data manager), Haruka Okamoto (data manager) and Toshihiro Yoshikawa (statistician).

\section{REFERENCES}

1. Hamaguchi $\mathrm{S}$, Yokoshiki $\mathrm{H}$, Kinugawa $\mathrm{S}$, Tsuchihashi-Makaya M, Yokota T, Takeshita A, JCARE-CARD Investigators, et al. Effects of atrial fibrillation on long-term outcomes in patients hospitalized for heart failure in Japan: a report from the Japanese Cardiac Registry of Heart Failure in Cardiology (JCARE-CARD). Circ J. 2009;73:2084-90.

2. Maisel WH, Stevenson LW. Atrial fibrillation in heart failure: epidemiology, pathophysiology, and rationale for therapy. Am J Cardiol. 2003;91:2D8D.

3. Sato N, Kajimoto K, Asai K, Mizuno M, Minami Y, Nagashima $M$, et al. Acute decompensated heart failure syndromes (ATTEND) registry. A prospective observational multicenter cohort study: rationale, design, and preliminary data. Am Heart J. 2010;159:949-55.

4. JCS Joint Working Group. Guidelines for pharmacotherapy of atrial fibrillation (JCS 2008): digest version. Circ J. 2010;74:2479-500.
5. Clark DM, Plumb VJ, Epstein AE, Kay GN. Hemodynamic effects of an irregular sequence of ventricular cycle lengths during atrial fibrillation. J Am Coll Cardiol. 1997;30:1039-45.

6. Wang TJ, Larson MG, Levy D, Vasan RS, Leip EP, Wolf PA, et al. Temporal relations of atrial fibrillation and congestive heart failure and their joint influence on mortality: the Framingham Heart Study. Circulation. 2003;107:2920-5.

7. Peters KG, Kienzle MG. Severe cardiomyopathy due to chronic rapidly conducted atrial fibrillation: complete recovery after restoration of sinus rhythm. Am J Med. 1988;85:242-4.

8. Grogan M, Smith HC, Gersh BJ, Wood DL. Left ventricular dysfunction due to atrial fibrillation in patients initially believed to have idiopathic dilated cardiomyopathy. Am J Cardiol. 1992;69:1570-3.

9. Fuster V, Rydén LE, Cannom DS, Crijns HJ, Curtis $\mathrm{AB}$, Ellenbogen $\mathrm{KA}$, et al. $2011 \mathrm{ACCF} / \mathrm{AHA} / \mathrm{HRS}$ focused updates incorporated into the ACC/AHA/ ESC 2006 Guidelines for the management of patients with atrial fibrillation: a report of the American College of Cardiology Foundation/ American Heart Association Task Force on Practice Guidelines developed in partnership with the European Society of Cardiology and in collaboration with the European Heart Rhythm Association and the Heart Rhythm Society. J Am Coll Cardiol. 2011;57:e101-98.

10. The Digitalis in Acute Atrial Fibrillation (DAAF) Trial Group. Intravenous digoxin in acute atrial fibrillation. Results of a randomized, placebocontrolled multicentre trial in 239 patients: the Digitalis in Acute Atrial Fibrillation (DAAF) Trial Group. Eur Heart J. 1997;18:649-54.

11. Shiroya T, Ichioka $Y$, Yoshida K, Nishijima K, Omawari N, Naka M, et al. Pharmacological studies of ONO-1101 as a beta-blocking agent with high beta 1 selectivity and ultra-short duration of action. Kiso To Rinsho. 1997;31:2913-23 (in Japanese).

12. Sakamoto A, Kitakaze M, Takamoto S, Namiki A, Kasanuki H, Hosoda S, JL-KNIGHT study group. Landiolol, an ultra-short-acting $\beta 1$-blocker, more effectively terminates atrial fibrillation than diltiazem after open heart surgery: prospective, multicenter, randomized, open-label study (JLKNIGHT study). Circ J. 2012;76:1097-101.

13. Hanada K, Higuma T, Nishizaki F, Sukekawa T, Yokota T, Yamada M, et al. Randomized study on the efficacy and safety of landiolol, an ultra-shortacting $\beta 1$-adrenergic blocker, in patients with acute myocardial infarction undergoing primary 
percutaneous coronary intervention. Circ J. 2012;76:439-45.

14. Kobayashi S, Susa T, Tanaka T, Murakami W, Fukuta $S$, Okuda $S$, et al. Low-dose $\beta$-blocker in combination with milrinone safely improves cardiac function and eliminates pulsus alternans in patients with acute decompensated heart failure. Circ J. 2012;76:1646-53.

15. Miwa Y, Ikeda T, Mera H, Miyakoshi M, Hoshida K, Yanagisawa R, et al. Effects of landiolol, an ultrashort-acting $\beta 1$-selective blocker, on electrical storm refractory to class III antiarrhythmic drugs. Circ J. 2010;74:856-63.

16. Nagai R, Kinugawa K, Inoue H, Atarashi H, Seino $Y$, Yamashita T, Shimizu W, et al. Urgent management of rapid heart rate in patients with atrial fibrillation/flutter and left ventricular dysfunction. Circ J. 2013;77:908-16.

17. Hunt SA, Abraham WT, Chin MH, Feldman AM, Francis GS, Ganiats TG, et al. 2009 Focused update incorporated into the ACC/AHA 2005 guidelines for the diagnosis and management of heart failure in adults: a report of the American College of Cardiology Foundation/American Heart Association Task Force on practice guidelines developed in collaboration with the International Society for Heart and Lung Transplantation. J Am Coll Cardiol. 2009;53:e1-90.

18. Adams KF Jr, Fonarow GC, Emerman CL, LeJemtel $\mathrm{TH}$, Costanzo MR, Abraham WT, et al. Characteristics and outcomes of patients hospitalized for heart failure in the United States: rationale, design, and preliminary observations from the first 100,000 cases in the Acute Decompensated Heart Failure National Registry (ADHERE). Am Heart J. 2005;149:209-16.

19. Hamaguchi S, Tsuchihashi-Makaya M, Kinugawa S, Yokota T, Ide T, Takeshita A, et al. Chronic kidney disease as an independent risk for long-term adverse outcomes in patients hospitalized with heart failure in Japan. Report from the Japanese Cardiac Registry of Heart Failure in Cardiology (JCARE-CARD). Circ J. 2009;73:1442-7.
20. Shiba N, Matsuki M, Takahashi J, Tada T, Watanabe J, Shimokawa H. Prognostic importance of chronic kidney disease in Japanese patients with chronic heart failure. Circ J. 2008;72:173-8.

21. Sackner-Bernstein JD, Skopicki HA, Aaronson KD. Risk of worsening renal function with nesiritide in patients with acutely decompensated heart failure. Circulation. 2005;111:1487-91.

22. Sackner-Bernstein JD, Kowalski M, Fox M, Aaronson K. Short-term risk of death after treatment with nesiritide for decompensated heart failure: a pooled analysis of randomized controlled trials. JAMA. 2005;293:1900-5.

23. O'Connor CM, Starling RC, Hernandez AF, Armstrong PW, Dickstein K, Hasselblad V, et al. Effect of nesiritide in patients with acute decompensated heart failure. $\mathrm{N}$ Engl J Med. 2011;365:32-43.

24. Teerlink JR, Cotter G, Davison BA, Felker GM, Filippatos G, Greenberg BH, et al. Serelaxin, recombinant human relaxin-2, for treatment of acute heart failure (RELAX-AHF): a randomised, placebo-controlled trial. Lancet. 2013;381:29-39.

25. Metra M, Cotter G, Davison BA, Felker GM, Filippatos G, Greenberg BH, et al. Effect of serelaxin on cardiac, renal, and hepatic biomarkers in the Relaxin in Acute Heart Failure (RELAX-AHF) development program: correlation with outcomes. JACC. 2013;61:196-206.

26. Motomura S, Hagihara A, Narumi Y, Hashimoto K. Time course of a new ultrashort-acting betaadrenoceptor-blocking drug, ONO-1101: comparison with those of esmolol and propranolol by using the canine isolated, bloodperfused heart preparations. J Cardiovasc Pharmacol. 1998;31:431-40.

27. Iguchi $S$, Iwamura $H$, Nishizaki $M$, Hayashi $A$, Senokuchi K, Kobayashi K, et al. Development of a highly cardioselective ultra short-acting betablocker, ONO-1101. Chem Pharm Bull. 1992;40:1462-9. 